\title{
A Simple a.c. Magnetic Susceptometer Using self-inductance Measurement of a Single Coil Mounted on a Cryostat Cold Head
}

\author{
Joonghoe Dho* \\ Dept. of Physics, Kyungpook National University, Daegu 702-701, Korea
}

(Received 21 August 2008, Received in final form 12 November 2008, Accepted 17 November 2008)

\begin{abstract}
A very simple a.c. magnetic susceptometer for use in a helium closed cycle cryostat is reported in this paper. This simple setup has only a single bobbin-less copper coil, instead of the primary coil and two secondary coils typically used in mutual-inductance types. The single bobbin-less copper coil is directly mounted on the cryostat cold head. A sample is attached on the inside wall of the copper coil using a thermal contact material and its a.c. magnetic susceptibility is obtained from the measurement of the self-inductance of the sample coil using an LCR meter or an impedance analyzer. Experimental details are described and illustrative measurements on magnetic and superconducting materials as a function of temperature are included. The performances and limitations of this simple a.c. magnetic suceptometer are also discussed.
\end{abstract}

Keywords : a.c. magnetic susceptibility, phase transition

\section{Introduction}

The measurement of alternating current (a.c.) magnetic susceptibility as a function of temperature is a straightforward way to characterize the magnetic property of a material. This has been frequently used to identify various phase transitions such as superconducting transition as well as magnetic phase transitions such as ferromagnetic, antiferromagnetic, and spin-glass transitions. In general, the measurement method of the a.c. magnetic susceptibility is based on the coaxial 'mutual-inductance' technique proposed by Hartshorn [1-9], where an a.c. magnetic field of approximately $10 \mathrm{~Hz}-10 \mathrm{kHz}$ is generated by a primary coil and the basic unit of the measurement circuit consists of two secondary coils which are oppositely wound to each other (Fig. 1(a)). In these types of a.c. magnetic susceptometers, the mutual inductance between primary and secondary coils is ideally zero in the absence of a sample and thus the measured e.m.f. across the secondary coils is also zero since the two secondary coils generate the same magnitude, yet have opposite sign voltages. If a magnetic sample is placed at the center of one of the secondary coils, a non-zero a.c. signal proportional to the a.c. magnetic susceptibility of the sample $\chi_{\mathrm{ac}}$ is observed.

*Corresponding author: Tel: $+82-53-950-7354$

Fax: +82-53-952-1739, e-mail: jhdho@knu.ac.kr
Recently, the measurement of a.c. magnetic susceptibility using commercial equipment is widely used at the research. However, such commercial equipment is very expensive so it is inconvenient for a general user performing routine research work at the laboratory. Accordingly, many researchers have manufactured a home-made a.c. magnetic susceptometer using a lock-in amplifier and a low temperature cryostat. Commercial equipment, as well as equipment reported in the literature, is mostly based on liquid helium cryostats, as seen in Fig. 1(a) [10-12]. However, in situations where daily liquid helium expenses and maintenance costs are not readily available, it is necessary to lower the temperature by means of a closed cycle cryostat which typically cools down a copper cold head in a vacuum. Therefore, the design of an a.c. magnetic susceptometer based on a closed cycle cryostat would be valuable because it would help to reduce research costs.

Until now, a number of researchers have reported attractive designs for an a.c. magnetic susceptometer based on a closed cycle cryostat [13-15]. T. Dumelow et al. reported a design of an a.c. magnetic susceptometer where the coil assembly was mounted on the cryostat cold head (Fig. 1(b)) [13]. Alternatively, Ph. Vanderbemden reported a simple type of a.c. magnetic susceptometer with only a single secondary coil instead of the typical two secondary oppositely wound coils (Fig. 1(c)) [14]. The primary coil was located on the outside cryostat and the single 
(a)

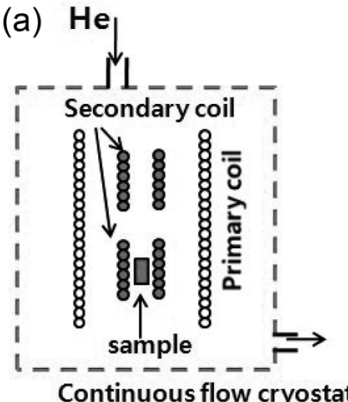

(c)

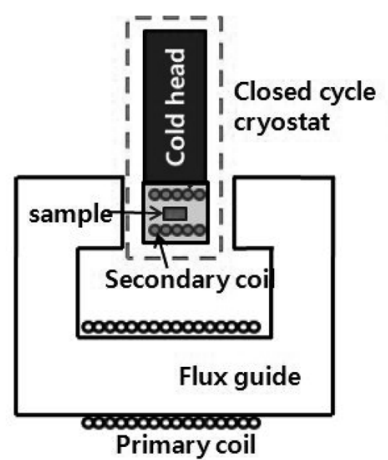

(b)

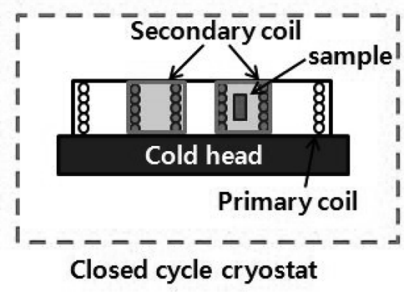

(d)

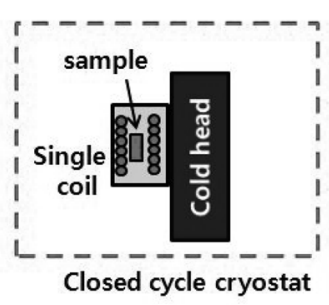

Fig. 1. Schematic diagrams of typical mutual-inductance type a.c. magnetic susceptometers with a primary coil and two oppositely wound secondary coils based on: (a) a continuous flow cryostat; and (b) a closed cycle cryostat, or; (c) with a primary coil and a single secondary coil based on a closed cycle cryostat. (d) A simple self-inductance type a.c. magnetic susceptometer based on a closed cycle cryostat.

secondary coil was mounted on a polycrystalline silicon rod which was in contact with the cryostat cold head and was covered with quartz glass to prohibit the eddy current effect. In this arrangement, placing a thermal contact material between the sample and the cold head allowed a minimum temperature of $\sim 20 \mathrm{~K}$ to be achieved. In a single coil type, the measured e.m.f. across the pick-up coil signal is proportional to the a.c. magnetic permeability $\mu_{\mathrm{m}}$ $=\mu_{0}\left(1+\chi_{\mathrm{ac}}\right)$. Therefore, a careful subtraction process should be carried out in order to find the amplitude of the a.c. magnetic susceptibility $\chi_{\mathrm{ac}}$. While this method may not be sensitive for very low susceptibility materials $\left(\left|\chi^{\prime}\right|<<1\right)$, it is still useful for measuring the a.c. magnetic property of materials such as superconductors and ferromagnets.

The simple design of an a.c. magnetic susceptometer based on a closed cycle cryostat would be useful and effective for performing routine research work in the laboratory. A very simple a.c. magnetic susceptometer based on a close cycle cryostat and the 'self-inductance' technique is reported in this paper. This simple setup consists of a single coil directly mounted on a cryostat cold head and the information on the a.c. magnetic sus-

ceptibility is obtained from the inductance measurement of a single coil using a typical LCR meter.

\section{Experimental Setup}

\subsection{Basic measurement technique}

Although the design of an a.c. magnetic susceptometer based on a closed cycle cryostat would be very useful, there are a number of problems associated with the detection coil assembly mounted on a cryostat cold head: (i) thermal conduction between the cryostat cold head and a sample needs to be sufficient to cool down the sample; (ii) the thermal contact material placed between the two coils must not affect the measured a.c. signal; (iii) if the coil assembly is metallic, it should be carefully designed to prohibit the eddy current effect; (iv) the temperature gradient between the sample and the temperature sensor should be considered; (v) the temperature change may cause thermal expansion or contraction in the coil assembly and an impendence change of the detection coils; (vi) compared with a capacitance measurement of a small capacitor, the inductance measurement of a coil can be largely affected by other impedance sources such as coating and fixing materials and the line capacitance in the compactly wound coil. Consequently, the background signal level will not be constant with the change of temperature, and a temperature dependent spurious signal may be added to the measured signal even in carefully designed detection coils.

It seems that any type of a.c. magnetic susceptometer is not completely free of the above problems. Accordingly, one can consider the simplified design of a single coil type and measure the change in the self-inductance of the coil both before and after the insertion of a sample in order to obtain information on the a.c. magnetic susceptibility. In the a.c. magnetic susceptometer described here, the sample coil attached on a cooper block is mounted on the cryostat cold head, and its self-inductance as a function of temperature or frequency is measured by an LCR meter or an impedance analyzer (Fig. 1(d)). The measured inductance value is subtracted from the self-inductance value for the empty coil.

The self-inductance $L$ for a solenoid type coil is defined as a differential of magnetic flux $\Phi$ with respect to the current $I$, and is therefore expressed by

$$
L=\frac{d}{d I} \Phi=\frac{d}{d I} B A=\frac{d}{d I} \mu H A=\frac{d}{d I} \mu_{0}\left(1+\chi_{\mathrm{ac}}\right) H A,
$$

where $\mu$ and $\mu_{0}$ are the magnetic permeabilities in a sample and in free space, respectively. The $\chi_{\mathrm{ac}}$ is the magnetic susceptibility of the sample, $H$ is the applied 
magnetic field, and $A$ is the area of coil. Therefore, the self-inductance change $\Delta L$ between before and after insertion of a sample is proportional to the a.c. magnetic susceptibility $\chi_{\text {ac, }}$ since

$$
\Delta L \propto \mu_{0}\left(1+\chi_{\mathrm{ac}}\right)-\mu_{0}=\mu_{0} \chi_{\mathrm{ac}} .
$$

In this method, the a.c. magnetic susceptibility is expressed in an arbitrary unit proportional to the inductance change $\Delta L$. The measurement method described here is probably suitable when using a closed cycle cryostat and an LCR meter or an impedance analyzer.

\subsection{Measurement system}

Enameled copper wires of $0.05 \mathrm{~mm}$ or $0.1 \mathrm{~mm}$ diameter were used for the bobbin-less sample coils. These coils either have a $3 \mathrm{~mm}$ inner diameter and a $3.5 \mathrm{~mm}$ length (870 turns) or a $6 \mathrm{~mm}$ inner diameter and a $9 \mathrm{~mm}$ length (670 turns). The bobbin-less sample coil was inserted into a hole of the cooper block with a small gap to prohibit the eddy current effect and was attached to the cooper block using an aluminum filled thermal conductive epoxy (Duralco ${ }^{\mathrm{TM}} 132$, resistivity $\sim 10^{6} \Omega \mathrm{cm}$ ). The coil assembly was directly mounted on the cold head of a helium closed cycle cryostat (Seongwoo Instrument Inc., cold head temperature $\sim 4 \mathrm{~K}$ ), and was connected to the external inductance measurement equipment (HP4284 LCR meter) through electrical feed-throughs. A sample was attached to an inside wall of the bobbin-less copper coil using GE varnish or Apiezon $\mathrm{N}$ grease for thermal conduction between the cold head and the sample. An applied a.c. current of $20 \mathrm{~Hz}-100 \mathrm{kHz}$ generates an oscillating magnetic field of 1-20 Oe in the sample coil. The a.c. magnetic field in the sample coil can be estimated from the constant a.c. current supplied by the LCR meter under the assumption of a solenoid type coil. The temperature was monitored by a Lakeshore temperature controller (LS331) using $\mathrm{Si}$ diode or Cernox ${ }^{\mathrm{TM}}$ sensors. All equipment is controlled by a personal computer via RS232 or IEEE488 interfaces.

\section{Results and Discussion}

In the small sample coil, the a.c. magnetic field may not be homogeneous since such a condition can only be achieved in an infinitely long solenoid type coil. In addition, the self-inductance value is not constant with frequency because of the frequency dependent characteristics of the electric circuit components (Fig. 2(a)). In addition, the measured self-inductance value of the sample coil is varied with the temperature, as shown in Fig. 2(b). Such a variation is probably due to complex impedance sources,

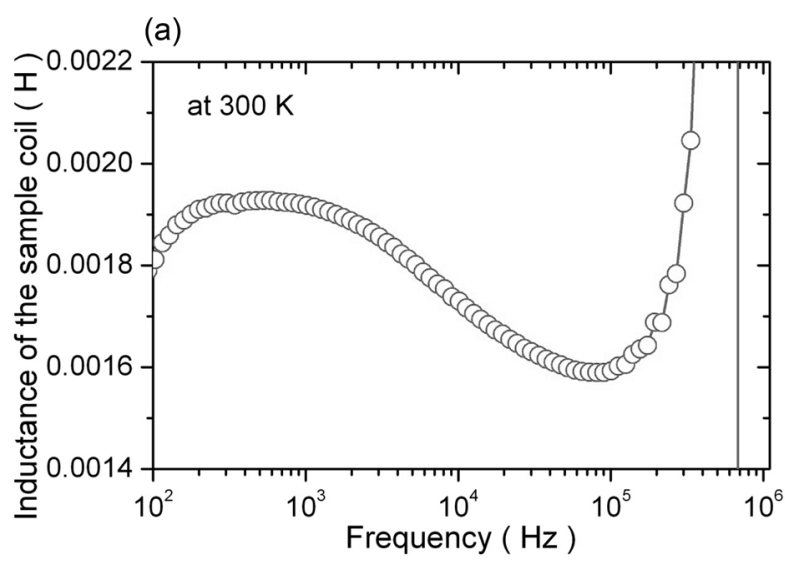

(b)

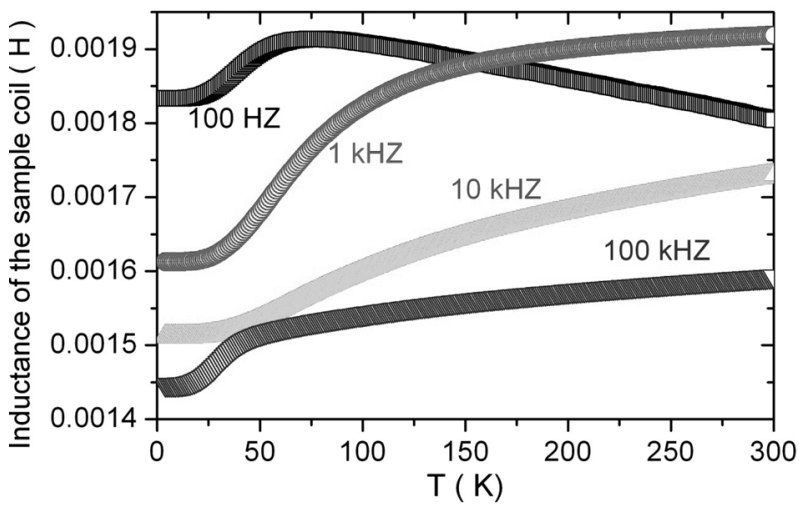

Fig. 2. (a) Frequency dependent self-inductance of the sample coil measured by an HP4284 LCR meter. (b) Temperature dependent self-inductance of the sample coil measured at 100 $\mathrm{Hz}, 1 \mathrm{kHz}, 10 \mathrm{kHz}$, and $100 \mathrm{kHz}$.

such as line capacitance, and coating and fixing materials in the compactly wound coil, which may cause a phase change of the electromagnetic wave and/or RLC resonance behavior. Thus the use of the simple a.c. magnetic susceptometer for absolute magnetic susceptibility measurements is definitely limited. However, this does not impede the use of the simple a.c. susceptometer for investigations of the temperature dependence of a.c. magnetic susceptibility $\chi_{\mathrm{ac}}(\mathrm{T})$, which can be expressed here in arbitrary units such as the self-inductance change $\Delta L(\mathrm{~T})$ of the coil after insertion of the sample. The $\chi_{\mathrm{ac}}(\mathrm{T})$ is frequently thought to be the most interesting information because it may be used to identify phase transitions in magnetic or superconducting materials.

Illustrative a.c. magnetic susceptibility measurements on magnetic and superconducting materials have been carried out using the simple setup based on a helium closed cycle cryostat. Fig. 3(a) shows the temperature dependent self-inductance change $\Delta L(\mathrm{~T})$ of the sample coil for a polycrystalline $\mathrm{La}_{0.46} \mathrm{Sr}_{0.54} \mathrm{Mn}_{0.98} \mathrm{Cr}_{0.02} \mathrm{O}_{3}$ sample at $10 \mathrm{kHz}$. For comparison, this is shown together with 
(a)

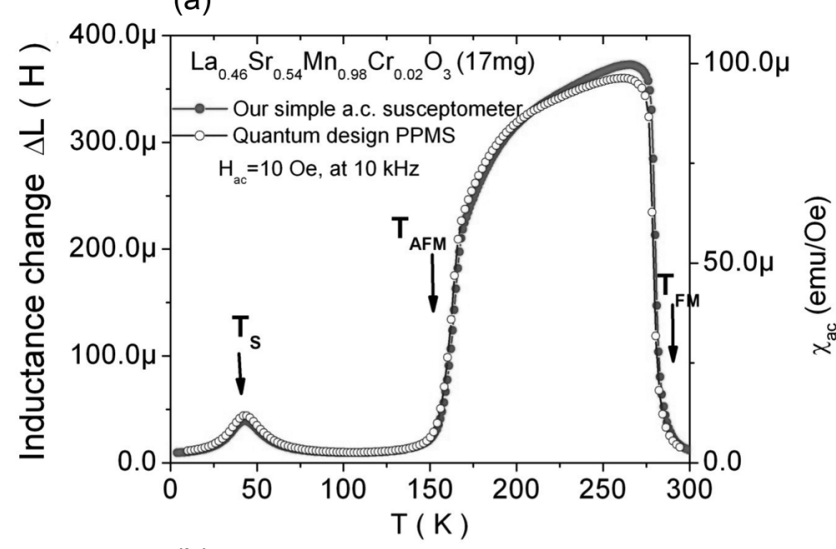

(b)

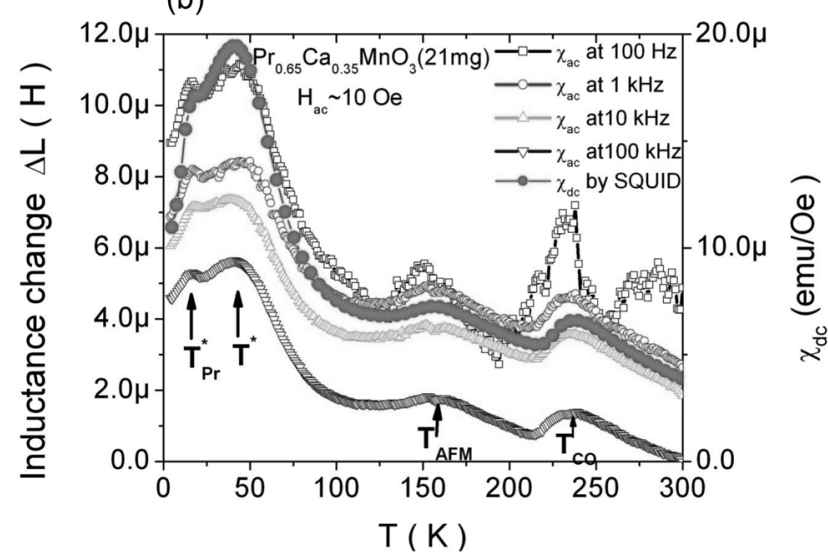

Fig. 3. (a) Temperature dependent inductance change $\Delta \mathrm{L}(\mathrm{T})$ for $\mathrm{La}_{0.46} \mathrm{Sr}_{0.54} \mathrm{Mn}_{0.98} \mathrm{Cr}_{0.02} \mathrm{O}_{3}$ measured at $10 \mathrm{kHz}$ using a simple self-inductance type a.c. magnetic susceptometer. For comparison, this is shown together with the a.c. magnetic susceptibility $\chi_{\mathrm{ac}}(\mathrm{T})$ measured by a commercial Qunatum design PPMS. (b) Temperature dependent inductance change $\Delta \mathrm{L}(\mathrm{T})$ for $\mathrm{Pr}_{0.65} \mathrm{Ca}_{0.35} \mathrm{Mn}_{2} \mathrm{O}_{3}$ measured at $100 \mathrm{~Hz}-10 \mathrm{kHz}$ using a simple self-inductance type a.c. magnetic susceptomter. For comparison, the zero-field-cooled d.c. magnetic susceptibility $\chi_{\mathrm{dcc}}(\mathrm{T})$ measured at 50 Oe using a commercial SQUID magnetometer (Qunatum design MPMS) is also shown.

the measured $\chi_{\mathrm{ac}}(\mathrm{T})$ data using a commercial a.c. magnetic susceptomter (Quantum design PPMS). The tested sample was synthesized by the conventional solid state reaction method described elsewhere [16]. It has been reported that $\mathrm{La}_{0.46} \mathrm{Sr}_{0.54} \mathrm{Mn}_{0.98} \mathrm{Cr}_{0.02} \mathrm{O}_{3}$ exhibits multiple magnetic phase transitions upon cooling: a paramagnetic (PM)-to-ferromagnetic (FM) transition at $\sim 280 \mathrm{~K}$, a ferromagnetic-to-antiferromagnetic (AFM) at $\sim 160 \mathrm{~K}$, and antiferromagnetic-to-spin glass-like transitions at $\sim 40 \mathrm{~K}$ [17]. The two curves obtained from both a.c. magnetic susceptometers are found to be practically identical although there is a subtle difference in the curvature with temperature.

In order to estimate the sensitivity of the simple a.c. magnetic susceptometer, the self-inductance change $\Delta L(\mathrm{~T})$ was measured for a low magnetic susceptibility material $\mathrm{Pr}_{0.65} \mathrm{Ca}_{0.35} \mathrm{MnO}_{3}$, which shows a charge order at $\mathrm{T}_{\mathrm{CO}} \sim$ $240 \mathrm{~K}$ and a paramagnetic-to-antiferromagnetic transition at $\mathrm{T}_{\mathrm{AFM}} \sim 170 \mathrm{~K}$. Fig. 3(b) shows the temperature dependent self-inductance change $\Delta L(\mathrm{~T})$ of the sample coil with a single crystalline $\operatorname{Pr}_{0.65} \mathrm{Ca}_{0.35} \mathrm{MnO}_{3}$ sample measured at $100 \mathrm{~Hz}-10 \mathrm{kHz}$. For comparison, this is shown together with the zero-field-cooled magnetizatic susceptibility measured at 50 Oe using a commercial SQUID magnetomter (Quantum design MPMS). The detail sample preparation method using an infrared optical furnace has been described elsewhere [18]. The measured $\Delta L(\mathrm{~T})$ curves clearly showed weak phase transitions such as charge ordering, antiferrromagnetic transition, and other weak magnetic transitions (marked by $\mathrm{T}^{*}$ and $\mathrm{T}_{\mathrm{Pr}_{\mathrm{r}}}{ }^{*}$ ) below $50 \mathrm{~K}$. The measured $\Delta L(\mathrm{~T})$ curve is slightly noisy at $100 \mathrm{~Hz}$, but was quite satisfactory for high frequencies. From the comparison with the commercial systems, the sensitivity of the simple a.c. magnetic susceptomter is estimated to be approximately at a $\sim 0.1 \mu \mathrm{emu} / \mathrm{Oe}$ level. The temperature dependent a.c. magnetic susceptibility measurement is often used to characterize the magnetic phase transition [19]. For several tests, the performance of the simple a.c. magnetic susceptomter was satisfactory for the characterization of phase transitions in similar magnetic materials.

Another illustrative measurement was performed for the superconducting $\mathrm{Nb}$ wire as a function of temperature. The $\Delta L(\mathrm{~T})$ data obtained at four different frequencies is shown in Fig. 4. The superconducting transition temperature $\left(T_{\mathrm{C}}\right)$ for the $\mathrm{Nb}$ sample (purity $99.8 \%$ ) is approximately $8 \mathrm{~K}$ at $100 \mathrm{~Hz}$, and it shows a slight decrease with

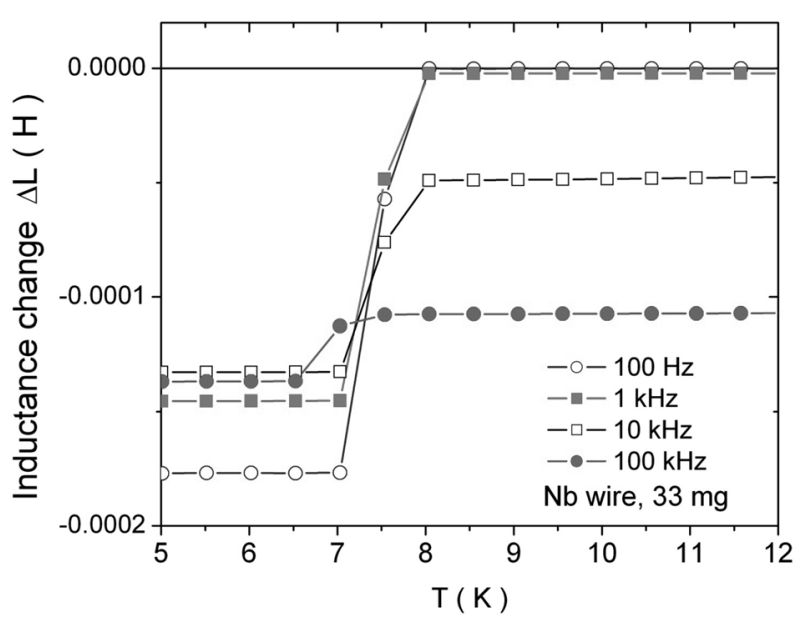

Fig. 4. Temperature dependent inductance change $\Delta \mathrm{L}(\mathrm{T})$ for the superconducting $\mathrm{Nb}$ wire measured at $100 \mathrm{~Hz}, 1 \mathrm{kHz}, 10$ $\mathrm{kHz}$, and $100 \mathrm{kHz}$ using a simple self-inductance type a.c. magnetic susceptometer based on a closed cycle cyrostat. 
the increase of frequency $\left(T_{\mathrm{C}} \sim 7.5 \mathrm{~K}\right.$ at $\left.100 \mathrm{kHz}\right)$. The measured $T_{\mathrm{C}}$ is slightly lower than the typical value of $T_{\mathrm{C}}$ $\sim 9 \mathrm{~K}$ for a high purity $\mathrm{Nb}$ wire, implying that the real temperature of the sample, attached on the inside wall of the sample coil using thermal contact materials, may be slightly different from that of the temperature sensor mounted on the cold head. It seems that the error in the real temperature of the sample increases with decreasing temperature because the thermal conductivity of the used thermal contact materials (GE varnish or Apiezon $\mathrm{N}$ grease) decreases slightly with decreasing temperature. The measured $T_{\mathrm{C}}$ suggests that the temperature error is about $\sim 1 \mathrm{~K}$ below $10 \mathrm{~K}$, and thus the system requires a delicate calibration for the real temperature of the sample in the inside coil.

\section{Conclusion}

A very simple experimental setup for the measurement of the temperature dependent a.c. magnetic susceptibility of a material was reported in this paper. The simple homemade a.c. magnetic susceptometer is based on a helium closed cycle cryostat working within the temperature range of $4 \mathrm{~K} \sim 320 \mathrm{~K}$ and a self-inductance measurement of a single coil which is directly mounted on the cryostat cold head. Therefore, this simple design is suitable when a closed cycle cryostat and an LCR meter, or an impedance analyzer, are used, and it is useful for the characterization of phase transitions in magnetic and superconducting materials.

\section{Acknowledgements}

The author would like to thank Mr. Donghoon Kim for his assistance. This work was supported by the Korea Research Foundation Grant funded by the Korean Government (MOEHRD, Basic Research Promotion Fund) (KRF2008-314-C00127) and the Korea Science and Engineer- ing Foundation (KOSEF) grant (R01-2006-000-10369-0).

\section{Rerefences}

[1] L. Hartshorn, J. Sci. Instrum. 2, 145 (1925).

[2] J. R. Owers-Bradley, W.-S. Zhou, and W. P. Halperin, Rev. Sci. Instrum. 52, 1106 (1981).

[3] R. B. Goldfarb and J. V. Minervini, Rev. Sci. Instrum. 55, 761 (1984).

[4] S. Ramakrishnan, S. Sundaram, R. S. Pandit, and G. Chandra, J. Phys. E: Sci. Instrum. 18, 650 (1985).

[5] L. Koszegi, M. Foldeaki, and R. A. Dunlap, Rev. Sci. Instrum, 62, 793 (1991).

[6] A. D. Hibbs, R. E. Sager, S. Kumar, J. E. McArthur, A. L. Singsaas, K. G. Jensen, M. A. Steindorf, T. A. Aukerman, and H. M. Schneider, Rev. Sci. Instrum. 65, 2644 (1994).

[7] R. R. de Souza and C. J. Magon, Rev. Sci. Instrum. 69, 431 (1998).

[8] C. P. Bidinosti and W. N. Hardy, Rev. Sci. Instrum. 71, 3816 (2000).

[9] M. I. Youssif, A. A. Bahgat, and I. A. Ali, Egypt. J. Sol. 23, 231 (2000).

[10] Couach, M. and Khoder, A. F. In: Magnetic susceptibility of superconductors and other spin systems, ed. Hein, R. A., Francavilla, T. L., Liebenberg, D. H. Plenum Press, New York (1992).

[11] Gömöry, F., Supercond. Sci. Technol. 10, 523 (1997).

[12] Nikolo, M., Am. J. Phys. 63, 57 (1995).

[13] T. Dumelow, M. M. Xavier Jr., F. A. O. Cabral, and C. Cheman, J. Magn. Magn. Mater. 226, 2063 (2001).

[14] Ph. Vanderbemden, Cryogenics 38, 839 (1998).

[15] M. Shahabuddin and N. S. Alzayed, Phys. Stat. Sol. c, 3, 3002 (2006).

[16] J. Dho, W. S. Kim, and N. H. Hur, Phys. Rev. Lett. 87, 187201 (2001).

[17] J. Dho, W. S. Kim, and N. H. Hur, Phys. Rev. Lett. 89, 072202 (2002).

[18] J. Dho and N. H. Hur, Solid State Comm. 140, 469 (2006).

[19] H. T. Kim, J. Magnetics 6(2), 57 (2001). 